\title{
SIMULAÇÃO DO DESLOCAMENTO DE POTÁSSIO EM COLUNAS VERTICAIS DE SOLO NÃO-SATURADO
}

\author{
JARBAS H. MIRANDA ${ }^{1}$, SERGIO N. DUARTE ${ }^{2}$, PAULO L. LIBARDI ${ }^{3}$, \\ MARCOS V. FOLEGATTI ${ }^{4}$
}

\begin{abstract}
RESUMO: O estudo do transporte de água e potássio em solo não-saturado é importante, tanto do ponto de vista do ambiente quanto do econômico. Assim sendo, o uso da modelagem computacional é importante, pois permite de maneira precisa e rápida o monitoramento do deslocamento de solutos, importante na prevenção de impactos ao ambiente. No presente trabalho, teve-se o objetivo de avaliar a simulação do deslocamento do íon potássio em colunas de solo não-saturado, utilizando o modelo MIDI, bem como apresentar a determinação dos parâmetros de transporte do íon potássio em um Latossolo Vermelho-Amarelo, fase arenosa. Concluiu-se que o modelo foi capaz de simular de maneira satisfatória o perfil de umidade e o deslocamento do íon potássio.
\end{abstract}

PALAVRAS-CHAVE: dinâmica de solutos, modelagem, curva de eluição.

\section{POTASSIUM DISPLACEMENT SIMULATION IN VERTICAL COLUMNS OF UNSATURATED SOIL}

\begin{abstract}
Water and solute transport studies in unsaturated soil are important for both economical and environmental points of view and, in this sense, it should be emphasized the increase of agricultural use of urban and industrial residues, to the water resources and fertilizers saving. Thus, the computational modeling use is important, because it allows the monitoring of solute displacement, necessary to the environmental impacts prevention in a precise and fast way. The main objective of the present work is to simulate the displacement of potassium ion in unsaturated soil columns, using the MIDI model, as well as to present transport parameters determination of the potassium ion in a Red Yellowish Latossol, sandy phase. The obtained results allowed concluding that the model was capable to adequately simulate the potassium ion displacement.
\end{abstract}

KEYWORDS: miscible displacement, modeling, breakthrough curve.

\section{INTRODUÇÃO}

O transporte simultâneo de água e de solutos em condições de fluxo não-saturado é objeto de interesse, variado e importante, pois a eficiência do uso da água e de produtos químicos nas produções agrícolas é por demais significativa, seja do ponto de vista do ambiente, seja econômico. Além disso, a possibilidade do uso de resíduos urbanos ou industriais, como forma de corrigir a deficiência nutricional de solos, tem sido considerada bastante promissora (MATOS et al., 1999).

O potássio, segundo MALAVOLTA (1980), é um macronutriente que tem caminhamento no solo, predominantemente pelo processo de difusão. Entretanto, alta parcela desse nutriente pode movimentar-se no solo por fluxo de massa. No Brasil, a aplicação de potássio às culturas é feita quase que totalmente no plantio e/ou cobertura, em aplicação direta ao solo ou via fertirrigação. Algumas vezes, a aplicação é complementada com adubações foliares (ZANINI, 1991).

\footnotetext{
${ }^{1}$ Eng ${ }^{\text {o }}$ Agrônomo, Prof. Dr., Departamento de Ciências Exatas, Escola Superior de Agricultura "Luiz de Queiroz" - ESALQ/USP, Av. Pádua Dias, 11, Piracicaba - SP, Fone: (0XX19) 3429.4283 ramal: 210, jhmirand@esalq.usp.br

${ }^{2}$ Prof. Dr. Departamento de Engenharia Rural, ESALQ/USP, snduarte@ esalq.usp.br

${ }^{3}$ Prof. Titular, Departamento de Ciências Exatas, ESALQ/USP, pllibard@esalq.usp.br

${ }^{4}$ Prof. Associado, Departamento de Engenharia Rural, ESALQ/USP, mvfolega@esalq.usp.br

Recebido pelo Conselho Editorial em: 7-6-2004

Aprovado pelo Conselho Editorial em: 20-9-2005
} 
ZANINI (1991) verificou que, de maneira geral, os locais de maiores concentrações de $\mathrm{K}^{+}$ coincidiram com os locais de maiores valores de umidade, evidenciando seu caminhamento por fluxo de massa, concordando com MALAVOLTA (1980), isto é, a distribuição de potássio no solo correlacionou-se com a distribuição de água no solo, indicando que se pode ter elevado controle da localização desse íon no solo em função da fertirrigação e da irrigação.

Para o sucesso da simulação e resolução das equações que permitem predizer o deslocamento de solutos no solo, é necessária uma determinação realista dos parâmetros de transporte que influenciam na relação solo-soluto. Os parâmetros mais importantes que devem ser determinados para esse fïm, são a velocidade da água no poro, os coeficientes de difusão e dispersão e o fator de retardamento, sendo esse último definido por VALOCCHI (1984) como sendo a relação entre a velocidade do soluto reativo e a velocidade média da água no poro. O coeficiente de difusão-dispersão é um parâmetro físico que considera a difusão iônica e a dispersão mecânica na solução do solo (GENUCHTEN \& WIERENGA, 1986).

Portanto, devido ao grande número de variáveis que envolvem o transporte de solutos no solo, a utilização de modelos de simulação torna-se desejável pela rapidez, precisão e por permitir que grande número de fatores e efeitos sejam contabilizados (MIRANDA, 2001). Vários modelos de lixiviação têm sido desenvolvidos, por exemplo, MOUSE (STEENHUIS et al., 1984), GLEAMS (KNISEL et al., 2000), HYDRUS (KOOL \& GENUCHTEN,1991), MIDI (MIRANDA, 2001). O modelo MIDI é um modelo unidimensional que consiste na resolução de equações diferenciais parciais de segunda ordem, ou seja, a equação diferencial geral que rege o movimento de água no solo e a equação diferencial difusão-convecção do transporte de solutos.

O presente trabalho traz como objetivo avaliar a simulação do deslocamento do íon potássio em colunas de solo não-saturado, utilizando-se do modelo MIDI, bem como apresentar a determinação dos parâmetros de transporte do íon potássio em um Latossolo Vermelho-Amarelo, fase arenosa.

\section{MATERIAL E MÉTODOS}

\section{Descrição do modelo}

O modelo computacional MIDI foi desenvolvido visando à simulação da dinâmica de solutos no solo por meio de soluções numéricas de equações diferenciais que descrevem esse transporte. $\mathrm{O}$ modelo matemático implementado consiste na solução de duas equações diferenciais parciais de segunda ordem, ou seja, a equação do movimento de água no solo que descreve a variação da distribuição de umidade e a equação do transporte de solutos que permite estimar as mudanças na concentração de solutos no solo com o tempo.

As taxas de transferência de água entre células contíguas são calculadas pela equação de DarcyBuckingham, que pode ser apresentada da seguinte forma:

$$
\mathrm{q}=-\mathrm{K}(\bar{\theta}) \frac{\Delta \phi \mathrm{t}}{\Delta \mathrm{Z}}
$$

em que,

q - fluxo de água entre as seções da coluna, $\mathrm{L} \mathrm{T}^{-1}$;

$\mathrm{K}(\theta)$ - condutividade hidráulica do solo no anel, função da umidade média $(\bar{\theta})$ do solo no anel, $\mathrm{LT}^{-1}$;

$\Delta \phi \mathrm{t}$ - diferença do potencial total da água no solo, $\mathrm{L}, \mathrm{e}$

$\Delta \mathrm{Z}$ - altura de cada anel da coluna, L.

O valor da condutividade hidráulica do solo não-saturado, ou seja, a função $K(\theta)$, é obtido a partir da condutividade hidráulica do solo saturado $\left(\mathrm{K}_{\mathrm{o}}\right)$ e dos parâmetros da curva de retenção de água 
no solo, de acordo com o modelo de MUALEM (1976) e GENUCHTEN (1980), apresentados na eq.(2).

$$
\mathrm{K}\left(\theta \left(=\mathrm{K}_{\mathrm{o}} \omega^{\ell}\left[1-\left(1-\omega^{1 / \mathrm{m}}\right) \mathrm{m}\right]^{2}\right.\right.
$$

em que,

$\mathrm{K}(\theta)$ - condutividade hidráulica do solo não-saturado, $\mathrm{L} \mathrm{T}^{-1}$;

Ko - condutividade hidráulica do solo saturado, $\mathrm{L} \mathrm{T}^{-1}$;

$\omega$ - saturação efetiva, adimensional;

$\ell$ - parâmetro empírico, considerado igual a 0,5 para a maioria dos solos, e

m - parâmetro da curva de retenção da água no solo, adimensional.

O valor da saturação efetiva é obtido pela eq.(3).

$$
\omega=\frac{\theta-\theta_{\mathrm{r}}}{\theta_{\mathrm{s}}-\theta_{\mathrm{r}}}
$$

em que,

$\theta$ - umidade volumétrica do solo na condição inicial, $\mathrm{L}^{3} \mathrm{~L}^{-3}$;

$\theta_{\mathrm{r}}$ - umidade volumétrica residual do solo, $\mathrm{L}^{3} \mathrm{~L}^{-3}$, e

$\theta_{\mathrm{S}}$ - umidade volumétrica do solo no ponto de saturação, $\mathrm{L}^{3} \mathrm{~L}^{-3}$.

Na superfície superior da primeira célula, em que está sendo aplicada a solução de potássio, o modelo considera que os valores da condutividade hidráulica e da umidade inicial correspondem ao ponto de saturação; em seguida, é determinado o potencial mátrico, fornecido pela eq.(4):

$$
\psi=\frac{\left(\frac{1}{\omega^{1 / m}}-1\right)^{1 / n}}{\alpha}
$$

em que,

$\alpha$ - parâmetro com dimensão igual ao inverso da tensão, $\mathrm{L}^{-1}, \mathrm{e}$

n - parâmetro da curva de retenção de água no solo, adimensional.

Com os valores obtidos do potencial mátrico adicionados ao potencial gravitacional de cada ponto, considerados na metade de cada célula da coluna, o modelo determina o gradiente de potencial entre as células e, assim, estima o fluxo de água entre as mesmas [eq.(1)], considerando os valores de $\mathrm{K}(\theta)$ médios entre as células.

De posse dos valores de fluxo de água e estando no primeiro intervalo de tempo de simulação, o modelo determina a variação de umidade que ocorreu em cada ponto da coluna, cujo valor é obtido pela eq.(5):

$$
\Delta \theta=\frac{\Delta \mathrm{q} \mathrm{A} \Delta \mathrm{T}}{\mathrm{V}}
$$

em que,

$\Delta \theta$ - variação de umidade nas células da coluna, $\mathrm{L}^{3} \mathrm{~L}^{-3}$;

$\Delta \mathrm{q}$ - diferença entre o fluxo que entrou e saiu da célula, $\mathrm{L} \mathrm{T}^{-1}$;

$\mathrm{A}$ - área da seção transversal da coluna, $\mathrm{L}^{2}$;

$\Delta \mathrm{T}$ - intervalo de tempo simulado, $\mathrm{T}, \mathrm{e}$

$\mathrm{V}$ - volume de cada anel da coluna, $\mathrm{L}^{3}$. 
Essa variação de umidade é acrescida a cada célula da coluna, quando o modelo iniciar o segundo intervalo de tempo de processamento e assim por diante, até atingir o tempo total de simulação.

Uma vez determinados os valores de fluxo de água entre as células da coluna, inicia-se a determinação da concentração de potássio em cada célula. O primeiro valor a ser obtido é o fluxo de massa do potássio, resultado do produto entre o fluxo de água, entre os anéis da coluna, já obtido, e a concentração de potássio da solução no solo que preenche a coluna.

Conforme já visto, o processamento do modelo baseia-se na discretização da coluna em células. A diferença de concentração $(\Delta C)$ utilizada para a determinação do fluxo de potássio por difusãodispersão é determinada dentro de cada simulação, no respectivo intervalo de tempo. Esse fluxo entre duas células contíguas da coluna é representado por $\mathrm{J}_{\mathrm{dh}}, \mathrm{M} \mathrm{L}^{-2} \mathrm{~T}^{-1}$, e apresentado na eq.(6):

$$
\mathrm{J}_{\mathrm{dh}}=\mathrm{D}(\bar{\theta}) \frac{\Delta \mathrm{C}}{\Delta \mathrm{Z}}
$$

Na eq.(6), D é o coeficiente de dispersão hidrodinâmica longitudinal, $\mathrm{L}^{2} \mathrm{~T}^{-1}$, dado por:

$$
\mathrm{D}=\mathrm{D}_{\mathrm{m}}+\mathrm{D}_{\text {disp }}
$$

em que,

$\mathrm{D}_{\mathrm{m}}$ - coeficiente de difusão do soluto no solo na umidade $\theta, \mathrm{L}^{2} \mathrm{~T}^{-1}$, e

$\mathrm{D}_{\text {disp }}$ - coeficiente de dispersão do soluto para o fluxo q e a umidade $\theta, \mathrm{L}^{2} \mathrm{~T}^{-1}$.

O coeficiente Dm é obtido pela eq.(8) a seguir:

$$
\mathrm{D}_{\mathrm{m}}=\left(\frac{\bar{\theta}^{10 / 3}}{\theta_{\mathrm{s}}^{2}}\right) \text { Do }
$$

em que,

$\bar{\theta}$ - umidade média entre células consecutivas, $\mathrm{L}^{3} \mathrm{~L}^{-3}$;

$\theta_{\mathrm{S}}$ - umidade volumétrica do solo no ponto de saturação, $\mathrm{L}^{3} \mathrm{~L}^{-3}$, e

Do - coeficiente de difusão molecular do soluto em água pura, $\mathrm{L}^{2} \mathrm{~T}^{-1}$.

O coeficiente $D_{\text {disp }}$ é calculado por:

$$
\mathrm{D}_{\text {disp }}=\frac{\lambda \mathrm{q}}{\bar{\theta}}
$$

em que,

$\lambda$ - dispersividade do meio poroso, parâmetro empírico que varia com a textura do solo e a distância coberta pelo soluto, $\mathrm{L}$.

O fluxo total de soluto entre as células da coluna é aditivo, ou seja, é o somatório do fluxo de massa e o fluxo de difusão-dispersão.

Para determinar a concentração do soluto em cada célula, é necessário determinar a quantidade de soluto que se movimenta, isto é, a quantidade de soluto que "entra" e "sai" em cada célula da coluna. Essas quantidades são calculadas pelo programa MIDI, realizando um balanço de massa. 


\section{Experimento em laboratório}

O trabalho de validação do modelo foi conduzido no laboratório de Física do Solo do Departamento de Engenharia Rural - ESALQ/USP, conforme esquema composto pelos componentes apresentados nas Figuras $1 \mathrm{e} 2$. As soluções foram preparadas em concentrações de $500 \mathrm{~kg} \mathrm{~m}^{-3}$ de potássio $(\mathrm{KCl})$, sendo o solo classificado como Latossolo Vermelho-Amarelo, fase arenosa.

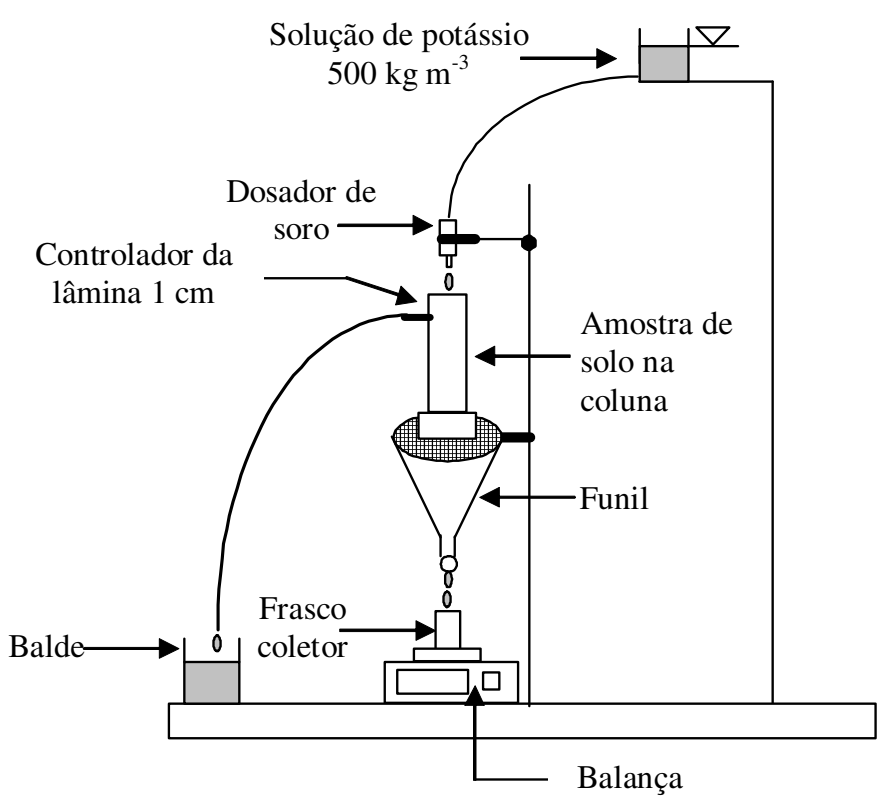

FIGURA 1. Esquema para a coleta de solução que atravessa a coluna de solo, para a elaboração da curva de eluição.

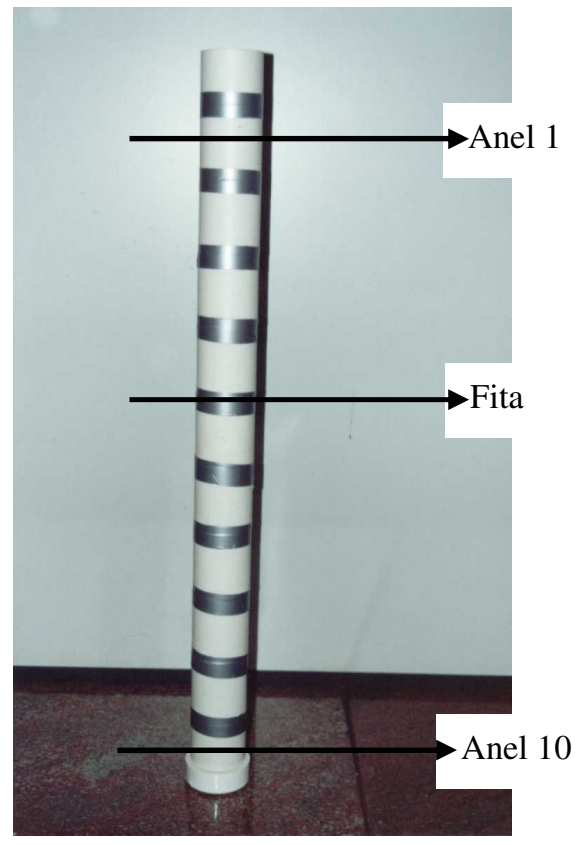

(A)

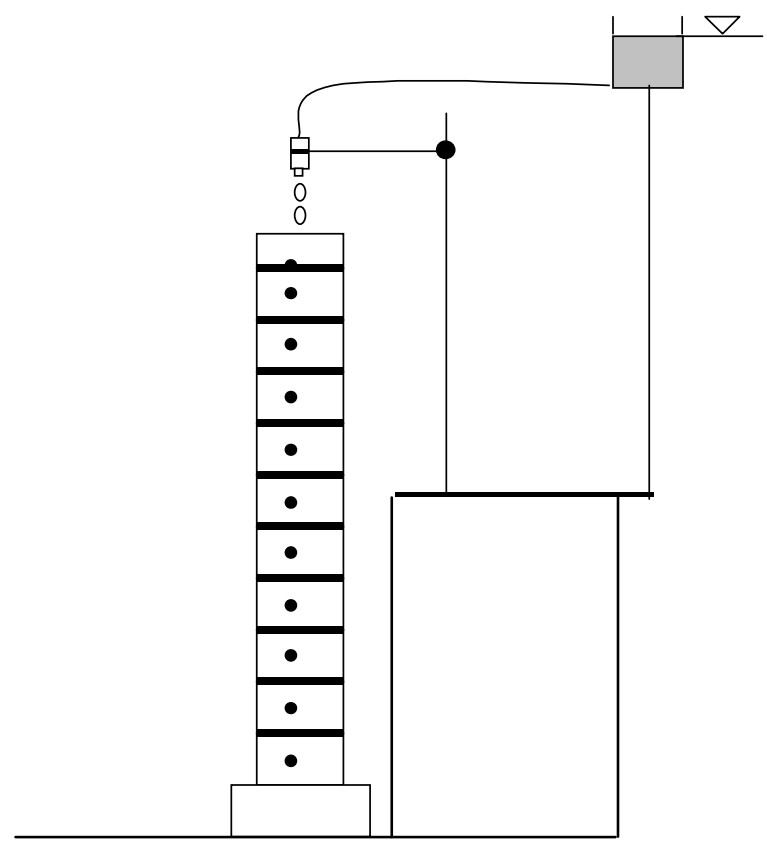

(B)

FIGURA 2. Coluna utilizada na aplicação da solução e determinação da concentração de potássio (A) e representação esquemática da aplicação da solução de potássio na coluna com um dosador (B). 
As análises físico-hídricas do solo apresentaram os seguintes resultados: $69,5 \%$ de areia; $12,0 \%$ de silte; $18,5 \%$ de argila; densidade do solo = 1,56 e densidade de partículas = 2,575; porosidade total do solo $=39,41 \%$ e condutividade hidráulica do solo saturado $=18,19 \mathrm{~cm} \mathrm{~h}^{-1}$. Os parâmetros da curva de retenção, ajustada segundo o modelo de GENUCHTEN (1980), foram: $\theta_{0}=0,0216 \mathrm{~cm}^{3} \mathrm{~cm}^{-3} ; \theta_{\mathrm{r}}=$ $0,162 \mathrm{~cm}^{3} \mathrm{~cm}^{-3} ; \theta_{\mathrm{s}}=0,443 \mathrm{~cm}^{3} \mathrm{~cm}^{-3} ; \alpha=0,0449 \mathrm{~cm}^{-1} ; \mathrm{n}=3,6732$, e $\mathrm{m}=0,1119$. A análise química foi determinada por amostras extraídas do material de solo do Latossolo Vermelho-Amarelo, fase arenosa (Tabela 1).

TABELA 1. Análise química da amostra do Latossolo Vermelho-Amarelo, fase arenosa, utilizado para o preenchimento da coluna.

\begin{tabular}{|c|c|c|c|c|c|c|c|c|c|}
\hline $\mathrm{pH}$ & M.O & $\mathrm{P}$ & $\mathrm{K}$ & $\mathrm{Ca}$ & $\mathrm{Mg}$ & $\mathrm{H}+\mathrm{Al}$ & SB & $\mathrm{T}$ & \multirow{2}{*}{$\begin{array}{c}\mathrm{V} \\
(\%)\end{array}$} \\
\hline $\mathrm{CaCl}_{2}$ & $\mathrm{~g} \mathrm{dm}^{-3}$ & $\mathrm{mg} \mathrm{dm}^{-3}$ & & & $--m$ & $\mathrm{l}_{\mathrm{c}} \mathrm{dm}^{-3}-$ & & & \\
\hline 4,6 & 21 & 5 & 1,8 & 24 & 9 & 31 & 34,8 & 65,8 & 53 \\
\hline
\end{tabular}

A coluna utilizada foi confeccionada em PVC e dividida em dez anéis, todos com dimensões de $5 \mathrm{~cm}$ de diâmetro e $7 \mathrm{~cm}$ de altura. O preenchimento foi feito com volumes iguais na tentativa de reduzir efeitos de fluxos preferenciais e da existência de camadas compactadas. Para impedir que houvesse vazamento da solução entre os anéis para a parte externa da coluna, utilizou-se de uma fita adesiva para a união dos anéis (Figura 2).

A aplicação das soluções foi feita por meio de um dosador de soro, para evitar variações na vazão de entrada na coluna de solo. Primeiramente, procedeu-se à regulagem do volume a ser aplicado por unidade de tempo, o qual ficou em torno de $150 \mathrm{~mL} \mathrm{~h}^{-1}$. Após essa regulagem do dosador, dava-se início à aplicação da solução.

Devido ao fato de a coluna ser de PVC, não era possível acompanhar a frente de molhamento, razão pela qual foi necessário fazer vários testes prévios para determinar o intervalo de tempo ideal, no qual a solução não atingia o final da coluna. O intervalo de tempo estipulado foi de $1 \mathrm{~h} 45$.

Após transcorrido o intervalo de tempo estipulado, desmontava-se a coluna e, à medida que isso era feito, coletava-se uma pequena amostra de cada anel para a determinação da umidade do solo na respectiva coordenada da coluna. O restante de solo de cada anel era utilizado para o preparo da pasta saturada, cujos valores de umidade ficavam próximos ao ponto de saturação. Após o período de 24 h, foram extraídas as soluções e conduzidas ao laboratório para a determinação das concentrações do íon potássio, realizada mediante a utilização de sensores de íons específicos.

Após as determinações da concentração do íon potássio no extrato de saturação, realizou-se, com três repetições, a correção dos valores obtidos para a umidade, antes do preparo da pasta de extração da solução [eq.(10)]:

$$
\mathrm{C}_{\text {ion corrigida }}=\frac{\mathrm{U}_{\text {pasta }} \mathrm{C}_{\text {ion }}}{\mathrm{U}_{\text {anel }}}
$$

em que,

$\mathrm{C}_{\text {ín corrigida }}$ - concentração do íon potássio corrigida em função da umidade, $\mathrm{g} \mathrm{cm}^{-3}$;

$\mathrm{U}_{\text {pasta }}$ - umidade com base em peso da pasta utilizada para a extração da solução do solo, $\mathrm{g} \mathrm{g}^{-1}$;

$\mathrm{C}_{\text {ín }}$ - concentração do íon (potássio) determinada na extração da solução, $\mathrm{g} \mathrm{cm}^{-3}, \mathrm{e}$

$\mathrm{U}_{\text {anel }}$ - umidade com base em peso do solo em cada anel da coluna, após o término da aplicação da solução, $\mathrm{g} \mathrm{g}^{-1}$. 


\section{RESULTADOS E DISCUSSÃO}

\section{Curvas de eluição}

A determinação dos parâmetros de transporte relativo ao solo utilizado foi feita mediante a utilização do modelo CXTFIT, desenvolvido pelo U.S. Salinity Laboratory - USDA - Riverside - CA, versão 2.1 (TORIDE, 1999).

Os valores obtidos dos parâmetros de transporte do potássio foram: velocidade da água nos poros $(V)=1,672 \mathrm{~cm} \mathrm{~min}^{-1}$; coeficiente de dispersão $(D)=2,703 \mathrm{~cm}^{2} \mathrm{~min}^{-1}$; fator de retardamento $(\mathrm{R})=$ 4,687; dispersividade $(\lambda)=1,61662 \mathrm{~cm}$; coeficiente de difusão (Do) a $25{ }^{\circ} \mathrm{C}=0,001188 \mathrm{~cm}^{2} \mathrm{~min}^{-1}$; concentração média inicial do soluto no solo $\left(\overline{\mathrm{C}}_{0}\right)=49,31 \mathrm{~kg} \mathrm{~m}^{-3}$, e concentração da solução aplicada de cada soluto $(\mathrm{Cs})=500 \mathrm{~kg} \mathrm{~m}^{-3}$. A representação gráfica dos valores experimentais, bem como dos ajustados pelo modelo CXTFIT, encontra-se na Figura 3.

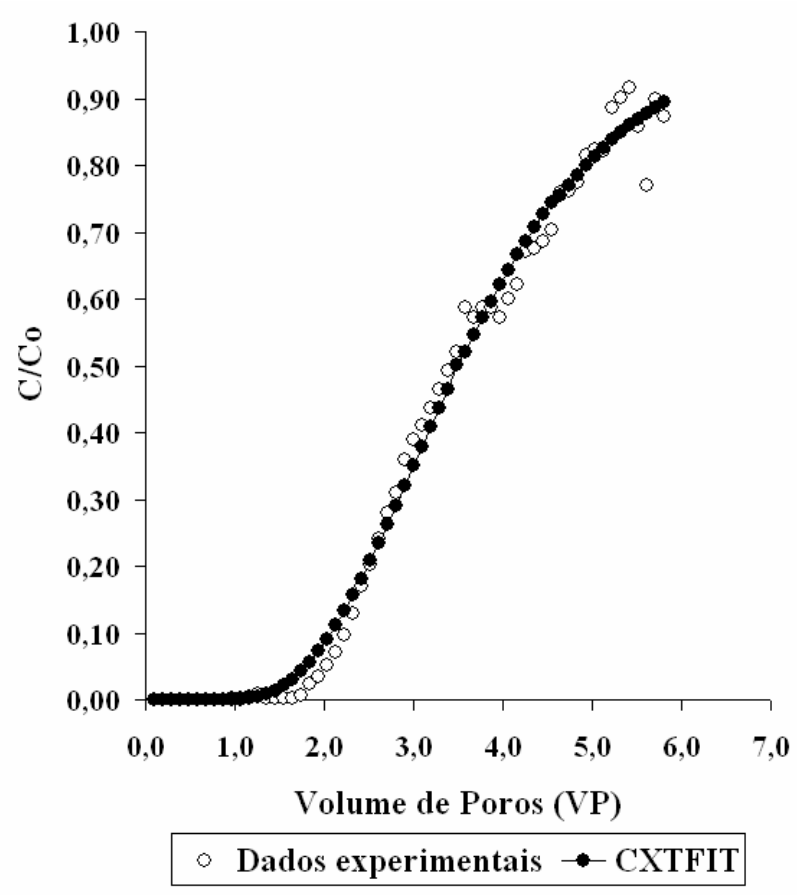

FIGURA 3. Curva de eluição obtida para o soluto de potássio no Latossolo Vermelho-Amarelo - Série "Sertãozinho".

\section{Validação da estimativa da umidade realizada pelo modelo}

Na Figura 4(A), apresentam-se os perfis de umidade medidos em três repetições e, na Figura 4(B), o perfil médio medido, além daquele simulado pelo modelo. Verifica-se que o modelo MIDI simulou, de maneira uniforme, o perfil de umidade resultante da aplicação da solução de potássio. As variações apresentadas são devidas, possivelmente, aos efeitos de preenchimento da coluna. 


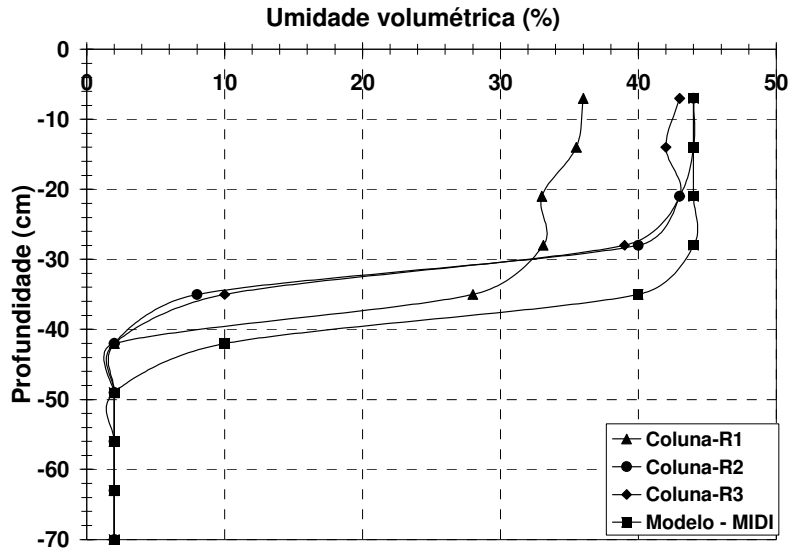

(A)

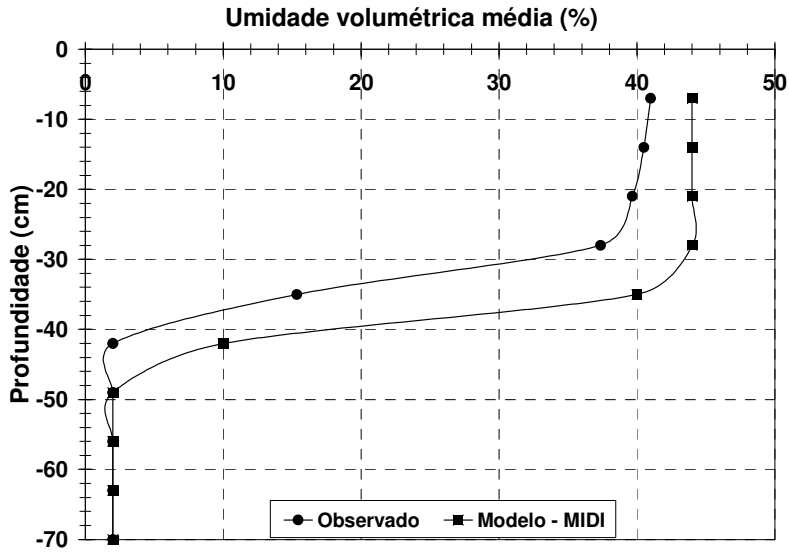

(B)

FIGURA 4 . Representação gráfica do perfil de umidade obtido na aplicação da solução de potássio (A) e média das três repetições mediante a aplicação da solução de potássio (B).

\section{Validação da estimativa da concentração de potássio realizada pelo modelo}

Em relação à simulação da concentração do íon potássio, pode-se observar maior variação entre as repetições, quando comparado com os dados de umidade. O solo que apresentava concentração inicial de 49,31 $\mathrm{kg} \mathrm{m}^{-3}$, ao término da aplicação da solução de potássio, revelou os valores médios que ficaram em torno de $300 \mathrm{~kg} \mathrm{~m}^{-3}$, nos anéis superiores. Há tendência, portanto, de maiores concentrações de potássio nas camadas superiores, em torno de $14 \mathrm{~cm}$, enquanto o molhamento se deu até cerca de $30 \mathrm{~cm}$. Esse resultado, possivelmente, deve-se ao fato de o potássio em solução ter sido adsorvido, de forma que a frente de molhamento que caminhou para profundidades maiores, provavelmente, apresentava menor concentração desse soluto (Figura 5).

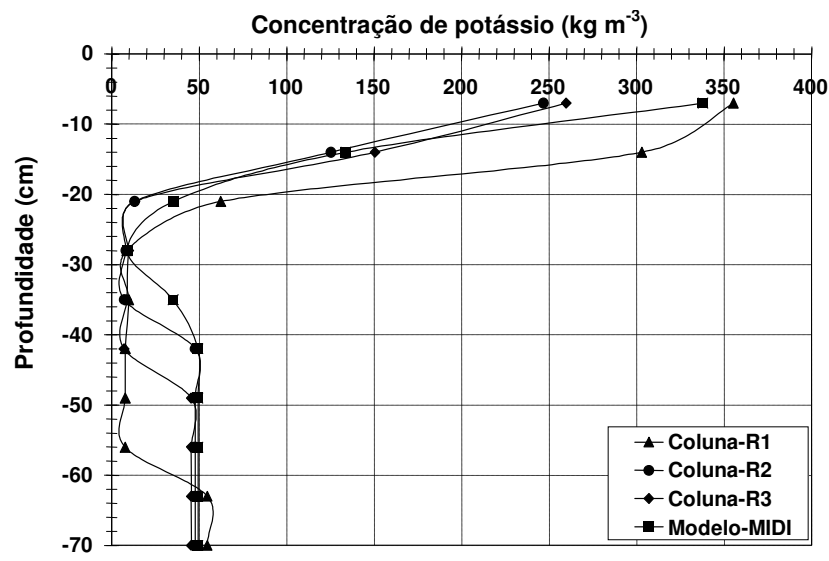

(A)

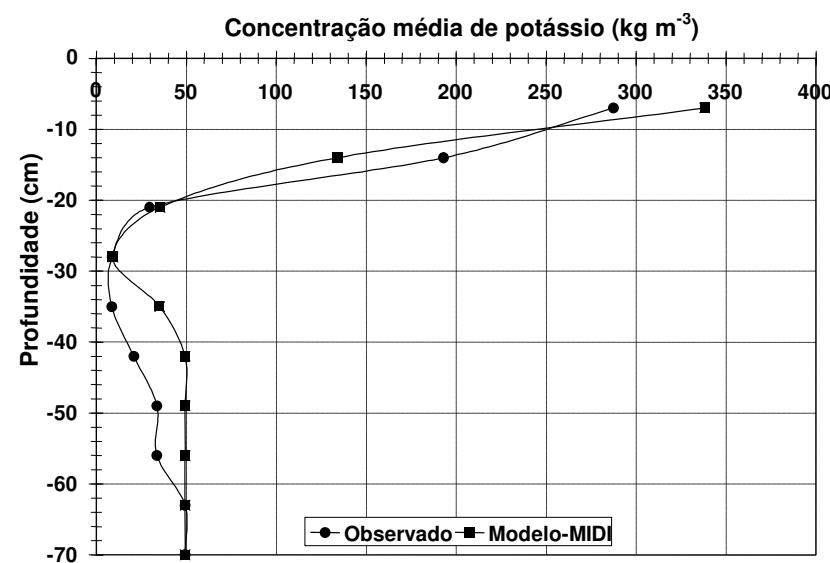

(B)

FIGURA 5. Representação gráfica do perfil de concentração do íon potássio obtido pela aplicação da solução de potássio (A) e média das três repetições mediante a aplicação da solução de potássio (B).

\section{CONCLUSÕES}

A simulação realizada pelo modelo MIDI foi capaz de prever, com bom ajuste, o perfil de umidade e o de deslocamento do íon potássio, incluindo o retardamento de movimentação desse íon em relação à frente de molhamento. Portanto, para que a simulação atinja padrões cada vez mais 
confiáveis, é necessário ressaltar a importância da determinação dos parâmetros de transporte, principalmente quando se trata da previsão do deslocamento de solutos no solo.

\section{REFERÊNCIAS}

GENUCHTEN, M.T. Van. A closed-form equation for predicting the hydraulic conductivity of unsaturated soils. Soil Science Society of America Journal, Madison, v.44, n.3, p.892-8, 1980.

GENUCHTEN, M.T. Van; WIERENGA, P.J. Solute dispersion coefficients and retardation factors. In: BLACK, C.A. (Ed.) Methods of soil analysis. Madison: Soil Science Society of America, 1986. p.1025-54. (American Society of Agronomy, 9)

KNISEL, W.G; DAVIS, F.M. GLEAMS - Groundwater loading effects of agricultural management systems. Version 3.0. User Manual. Tifton: U.S. Department of Agriculture, Agricultural Research Service, Southeast Watershed Research Laboratory, 2000. 194 p.

KOOL, J.B.; GENUCHTEN, M.T. Van. HYDRUS one-dimensional variably saturated flow and transport model, including hysteresis and root water uptake. Riverside: U.S. Salinity Laboratory USDA-ARS, 1991. $101 \mathrm{p}$.

MALAVOLTA, E. Potássio, magnésio e enxofre nos solos e culturas brasileiras. 2.ed. Piracicaba: Instituto Internacional de Pesquisa da Potassa, 1980. 91 p. (Boletim Técnico, 4)

MATOS, A.T.; COSTA, L.M.; FONTES, M.P.F.; MARTINEZ, M.A. Retardation factors and the dispersion-diffusion coefficients of $\mathrm{Zn}, \mathrm{Cd}, \mathrm{Cu}$ and $\mathrm{Pb}$ in soils from Viçosa-MG, Brazil. Transactions of the ASAE, St. Joseph, v.42, n.4, p.903-10, 1999.

MIRANDA, J.H. Modelo para simulação da dinâmica de nitrato em colunas verticais de solo não saturado. 2001. 79 f. Tese (Doutorado em Irrigação e Drenagem) - Escola Superior de Agricultura “Luiz de Queiroz", Universidade de São Paulo, Piracicaba, 2001.

MUALEM, Y. A new model for predicting the hydraulic conductivity of unsaturated porous media. Water Resources Research, New York, v.12, n.3, p.513-22, 1976.

STEENHUIS, T.S.; HUGHES, H.B.F.; PACENKA, S.; GROSS, M. MOUSE User's Manual. New York: Northeast Regional Agricultural Engineering Service, Cornell University, 1984. 44 p.

TORIDE, N.; LEIJ, F.J.; GENUCHTEN, M.T. Van. The CXTFIT Code for Estimating Transport Parameters from Laboratory or Field Tracer Experiments. Version 2.0. Riverside: U.S. Salinity Laboratory, 1999. 119 p. (Research Report, 137)

VALOCCHI, A.J. Describing the transport of ion-exchanging contaminants using an effective Kd approach. Water Resources Research, St. Louis, v.20, n.4, p.499-503, 1984.

ZANINI, J.R. Distribuição de água e do íon $\mathrm{K}^{+}$no solo, aplicados por fertirrigação em gotejamento. II

- Teores de $\mathrm{K}^{+}$no bulbo molhado. ITEM - Irrigação e Tecnologia Moderna, Brasília, v.46, n.1, p.24$38,1991$. 